figures, an amply sufficient accuracy. The principal merit of the graphs, however, is that the investigator can judge how the tentative configuration should be modified in order to make its structure-factor correspond to the observed value for each reflection, since the effect of moving an atom in a given direction and by a given distance is shown by the graph. The advantage of being able to do this will be appreciated by anyone accustomed to X-ray analysis.

By moving the origin from centre to corner of a small cell, the same graphs give values of $\sin (2 \pi h x / a) \sin (2 \pi k y / b)$. A single set of graphs, in fact, covers all cases of monoclinic and orthorhombic crystals for which the projection has a rectangular outline and a centre of inversion.

The advantage of the graphs is even greater in projections of tetragonal and hexagonal symmetry, when the atoms may be in groups of eight or twelve. The (230) graph for the tetragonal complex of Fig. $2 a$ is shown in Fig. $2 b$. Such a graph considerably facilitates the adjustment of atomic positions. There are plateaux, for example, where the contribution of an atom varies very slowly, so that atoms in these regions give an approximately known contribution and attention may be concentrated on other atoms in 'sensitive' positions.

Trial has shown that analysis can be much more rapidly carried out with the graphs than by processes of calculation.

$$
\begin{gathered}
\text { Physical Laboratories, } \\
\text { University, } \\
\text { Manchester. } \\
\text { July } 27 .
\end{gathered}
$$

W. L. BragG.

\section{Molecular State of Proteins in Mixtures and Con- centrated Solutions}

By means of the Svedberg high-speed ultracentrifuge it is possible to analyse mixtures of different molecular species, since the sedimentation involves a partial separation of the different sizes present. The concentration of the components may therefore be calculated from the sedimentation diagram. But during the study of protein mixtures in the ultra. centrifuge it has often been observed that the concentration of the different sedimenting molecules thus calculated were not the same as those determined analytically. Generally the apparent concentration of the faster sedimenting molecule was smaller, when calculated from the sedimentation diagram, whereas it was higher for the slower sedimenting molecule. McFarlane ${ }^{1}$, who first studied the problem on some artificial serum mixtures, thought that the effect was due to some specific interaction between the albumin and the globulin. Later, R. A. Kekwick and I studied some mixtures of serumalbumin and lactoglobulin. In this case serumalbumin, the faster sedimenting molecule, is found in too low concentration, and the lactoglobulin is found in too high concentration. From these experiments I concluded that the phenomenon was caused by some medium effect produced by the slower sedimenting protein molecules on the faster sedimenting ones, and that the effect was due to a real dissociation of the faster sedimenting molecules. If this is true, one would expect that it would be possible to produce the effect by means of lower molecular substances related to proteins, such as amino acids, polypeptides and protamines.
For the first two groups of these substances there was a measurable although very small effect. In the case of the protamine studied (clupein) the effect was very strong with serumalbumin, where it gave rise to new molecules with $s_{20}$ (= sedimentation constant) of about $1 \times 10^{-13}$ besides the ones with the normal $s_{20}\left(4.5 \times 10^{-13}\right)$. If we call the serumalbumin molecules with the normal $s_{20}, A$, and those of the dissociated serumalbumin, $B$, then the concentration ratio between these as calculated from the sedimentation diagram was : for a $5 \cdot 3$ per cent clupein solution $B / A=1.58$, for a 2.65 per cent solution $B / A=0.90$ and for a $1 \cdot 1$ per cent solution $B / A=0 \cdot 36$. From these figures it is seen that the effect is by no means negligible since the dissociation product of serumalbumin in the more concentrated clupein solution is present in higher concentration than the undissociated serumalbumin. It is also very remarkable that all the sedimentation diagrams show only the presence of molecules with $s_{20}=1 \times 10^{-13}$ and $s_{20}=4.5 \times 10^{-13}$ (besides a small amount of some association products); this probably indicates that the dissociation process always gives rise to molecules of the same size (perhaps one eighth of the original value). Some experiments with hæmoglobin show that this protein too is dissociated in the presence of other proteins and clupein, but here the sedimentation constant indicates that the dissociation at first gives molecules of half the original size, independent of whether lactoglobulin or clupein is used.

As the effect seems to be of a more general nature (whether it is a dielectric or chemical effect or a combined effect is at present impossible to decide) it is to be expected that it may also take place in a more concentrated solution of a uniform protein, and these solutions should therefore give a decreased molecular weight with increasing protein concentration. This may at least partly explain the fact that several investigations show that the proteins are behaving osmotically abnormally in that the ratio $\pi / c(\pi=$ osmotic pressure, $c=$ concentration of protein) increases with increasing protein concentration, as it should do if the molecular weight is not constant, but decreases with increasing concentration.

Information about this effect may be of value in explaining some of the biological processes, where we are dealing with more or less concentrated protein solutions.

Further details will be given in a forthcoming paper in the Biochemical Journal.

Laboratory of Physical Chemistry, University of Uppsala,

Sweden.

July 26.

${ }^{1}$ See, for example, A. S. McFarlane : Biochem. J., 29, 407 (1935).

\section{Effect of Phenylacetic Acid on the Growth of Tomato Plants}

MUCH interest has been taken in the action on the growth of plants of various organic substances which has been described by the workers at the Boyce Thompson Institute ${ }^{1,2}$. They apply these substances in lanolin preparations to plants, or as a water solution added to the soil or injected into the stem; the main responses of the treated plants being 